\title{
Fourier space imaging of light localization at a photonic band-edge located below the light cone
}

\author{
N. Le Thomas and R. Houdré \\ Institut de Photonique et d'Electronique Quantique, Ecole Polytechnique Fédérale de Lausanne (EPFL), \\ Station 3, CH-1015 Lausanne, Switzerland \\ D. M. Beggs and T. F. Krauss \\ SUPA, School of Physics and Astronomy, University of St. Andrews, St. Andrews, Fife KY16 9SS, United Kingdom
}

(Received 3 December 2008; published 20 January 2009)

\begin{abstract}
We observe light localization in a two-dimensional geometry, induced by residual disorder at a photonic band edge located below the light cone. The combination of a spectrally selective illumination and a grating assisted $k$-space imaging technique allows us to image the equifrequency surfaces associated with such a photonic band, with high accuracy and without aberrations. Thanks to this approach, the impact of the nonideal nature of real planar photonic crystals on the propagation properties of the Bloch wave at the band edge is decorrelated from the contribution of the intrinsic out-of-plane losses. As a by-product, our result demonstrates an immersion free effective numerical aperture as high as 3.5 in $k$ space.
\end{abstract}

DOI: 10.1103/PhysRevB.79.033305

PACS number(s): 42.30.Kq, 42.25.Bs, 42.70.Qs, 72.15.Rn

The propagation properties of waves are governed by the dispersion relation $\omega(\vec{k})$ between their angular frequency $\omega$ and their wave vector $\vec{k}=\left(k_{x}, k_{y}, k_{z}\right)$. In ideal two-dimensional (2D) periodic structures, the dispersion diagram $(\vec{k}, \omega)$ is composed of frequency bands that are separated by band gaps when the Bragg condition is satisfied. Near a band gap, an ideal band evolves toward a singular point and the curvature of the band toward this point implies a progressive slowing down of the speed of the corresponding waves down to a complete stop. Such a behavior at the band edge is particularly appealing for light waves in order to delay light propagation or to enhance nonlinear interaction with matter. At frequency corresponding to the extremum of the band, the wave is expected to be "frozen" and delocalized uniformly over all the structure.

However, in real photonic structures, such as planar photonic crystal slabs where the light is controlled at the subwavelength scale, ${ }^{1}$ two major effects affect this ideal behavior: the intrinsic out-of-plane losses and the residual structural disorder. Both phenomena impact on the curvature of the band and limit the range of propagating slow light. ${ }^{2}$ Intrinsic out-of-plane losses result from the coupling of the propagating modes in the planar structure with the free space mode. This coupling occurs if the condition $k_{x}^{2}+k_{y}^{2} \leq(\omega / c)^{2}$ is satisfied, where $\left(k_{x}, k_{y}\right)$ are the components of the in-plane wave vector and $c$ is the speed of light. In the dispersion diagram $\left(k_{x}, k_{y}, \omega\right)$, this condition defines a cone, called the light cone: the inner and outer parts of the light cone correspond, respectively, to waves which can propagate in free space and to evanescent waves localized near the slab.

The dispersion band diagram of planar photonic crystals can be engineered in order to be entirely located below the light cone over wide frequency ranges. As a result, intrinsic out-of-plane losses, i.e., intrinsic dissipation, are zero, and only the residual disorder, which we expect to lead to spatial localization of the waves, affects the propagation properties. In such a situation, per nature, no information escapes from the structures; therefore, the investigation of the band edge of the $2 \mathrm{D}$ photonic band is challenging.
Twenty years ago, in a pioneering work Zengerle $^{3}$ mapped the dispersion band diagram of periodic planar waveguides whose modes were located below the light cone. To excite these modes, he used a prism coupler and to determine the dispersion curve, he measured the direction of propagation of the excited waves that were scattered "owing to the more or less homogeneously distributed imperfections present everywhere" in the structure. However, such an approach which relies on noncontrolled light scattering does not enable per principle to reveal the impact of the disorder on the dispersion curve. In particular, no data near the singular point of the band edge in the $(\vec{k}, \omega)$ diagram were presented.

In this Brief Report, we use a 2D probe grating in order to investigate, in the optical Fourier space, the impact of the residual disorder on a photonic band edge. As a result, we highlight a disorder induced localization effect, in a 2D geometry, near the top band edge of the first photonic band of a square lattice $\mathrm{PhC}$. By observing the dispersion properties of well defined $\mathrm{PhC}$ structures directly in the back-focal plane of a collecting microscope objective, our results also demonstrate the achievement of an effective numerical aperture as high as 3.5.

In optical measurement, the bandwidth of the transfer function between the object and the detector is defined by the entrance pupil of the collecting lens. When the collecting lens is a microscope objective designed to satisfy the Abbe condition, the radius $k_{p}$ of the entrance pupil is given by $k_{p}$ $=(2 \pi / \lambda) \times \mathrm{NA}$, where NA is the numerical aperture of the microscope objective, and $\lambda$ the wavelength of the field in free space. The waves propagating in the planar structure are collected by the instrument if their transverse spatial frequency lies inside the entrance pupil of the instrument. The theoretical maximum limit of the radius of the pupil in free space is given by the radius of the light cone $k_{\mathrm{lc}}=(2 \pi / \lambda)$ $=\omega / c$, which corresponds to NA=1. One approach to overcome this limit is to use probe gratings to transfer the information in the pupil via a coherent scattering process. ${ }^{4-6}$ This technique is appealing for its parallel processing of the infor- 

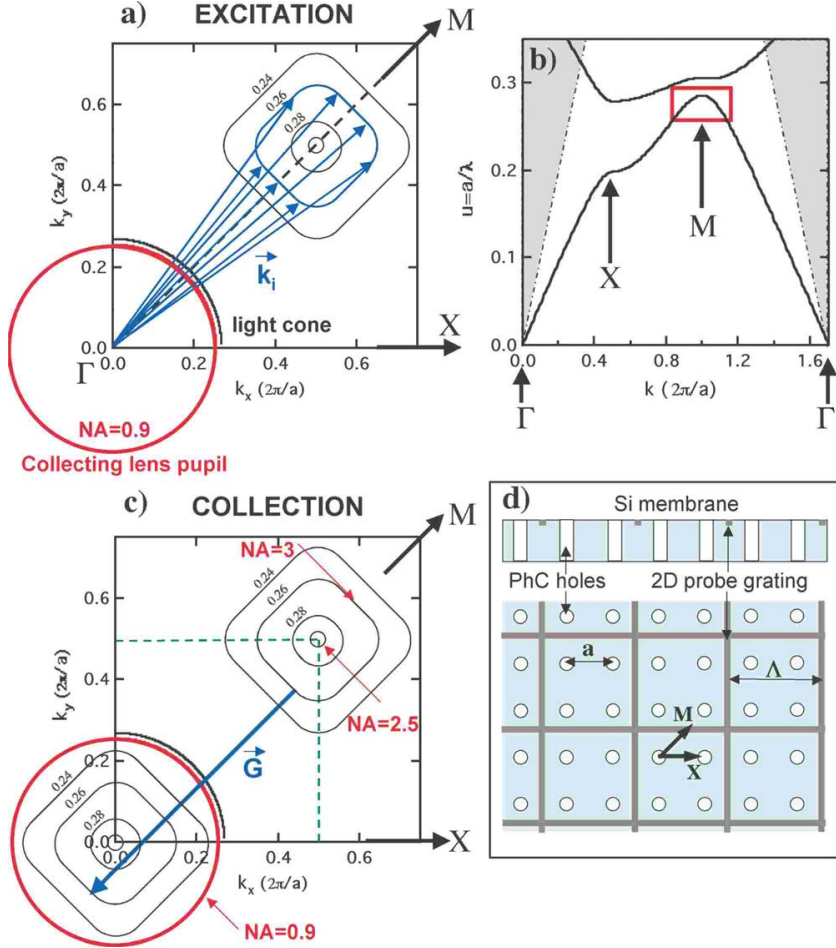

FIG. 1. (Color online) Principle of the 2D grating assisted superresolution in $k$ space. (a) Equifrequency surfaces of a square lattice $\mathrm{PhC}$. The excitation of a given EFS along the $\Gamma M$ direction is provided by the set of wave vectors $\vec{k}$ depicted as blue (dark gray) arrows. (b) Energy band diagram along the $\Gamma-X-M-\Gamma$ path. The red (dark gray) rectangle at the $M$ point highlights the region of interest. Shaded region: light cone. (c) Collection of the information via the folding of the EFS into the light cone. (d) Schematic side and top views of the $\mathrm{PhC}$ structure with a $2 \mathrm{D}$ probe grating (gray lines). mation. In addition, it can be combined with standard microscope objectives and it allows aberration free imaging in $k$ space.

In Fig. 1, we describe the principle of the grating based far-field imaging of a 2D photonic band located below the light cone. As an example, we have chosen the first photonic band of a PhC square lattice tile operating near the $M$ point for a TE polarized light and with lattice constant $a$. The equifrequency surfaces (EFS), which are the projections of the photonic band in the $\left(k_{x}, k_{y}\right)$ plane for a given frequency $\omega$, are located at the boundaries $\left(k_{x}= \pm 0.5 \frac{2 \pi}{a}, k_{y}\right.$ $\left.= \pm 0.5 \frac{2 \pi}{a}\right)$ of the first Brillouin zone in the wavelength range of interest $1.48 \mu \mathrm{m} \leq \lambda \leq 1.64 \mu \mathrm{m}$. In all imaging acquisition techniques, both the excitation and collection processes should be considered. The spectral selection is essential to recover the physics carried by the EFS, so we have used a narrow linewidth $(\Delta \lambda=0.5 \mathrm{pm})$ tunable laser.

The control of the incident wave vector excitation is provided by a narrow $(\sim 2 \mu \mathrm{m}$ wide and $0.23 \mu \mathrm{m}$ thick $)$ integrated access waveguide at the entrance of the PhC tile [see Fig. 2(a)]. The diffraction of the beam at the interface between the access waveguide and the $\mathrm{PhC}$ tile along the $\Gamma M$ direction ensures the excitation of all the in-plane wave vectors that form the selected EFS, as illustrated in Fig. 1(a). To measure the EFS located below the light cone in the far field, the information about the EFS that is carried by the Bloch waves excited in the $\mathrm{PhC}$ structure has to be transferred in the pupil of the microscope objective. The pupil $(\mathrm{NA}=0.9)$ is represented by the red circle (dark gray) in Fig. 1 and is very close to the light cone. A properly designed 2D probe grating perfectly transfers an image of the EFS into the far field, i.e., without deforming the shape of the EFS. The process relies on the conservation of the wave vectors during the scattering process: $\vec{k}_{\text {out }}=\vec{k}_{\text {in }}+\vec{G}$, with $\vec{k}_{\text {in }}$ one of the wave vectors asso-
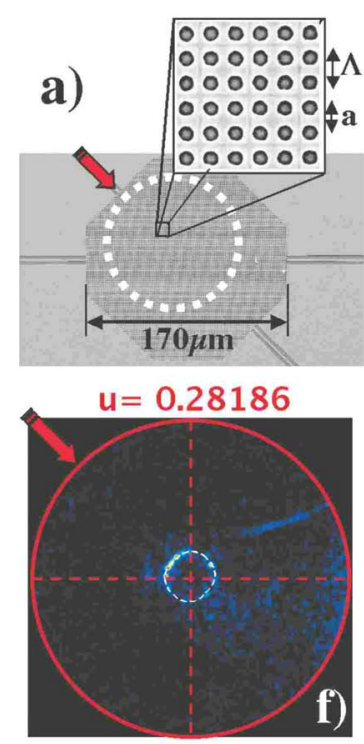

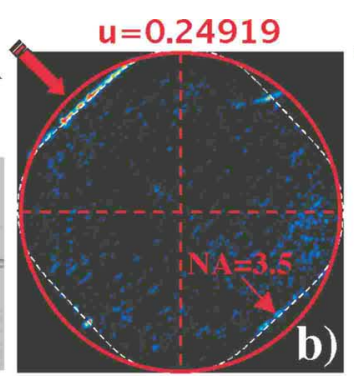

$\mathrm{u}=0.28278$

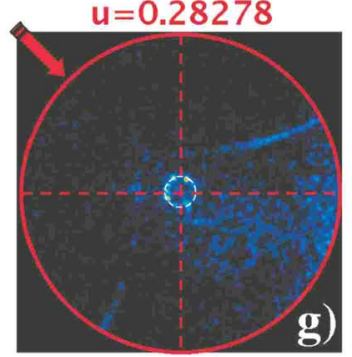

$\mathrm{u}=0.25361$

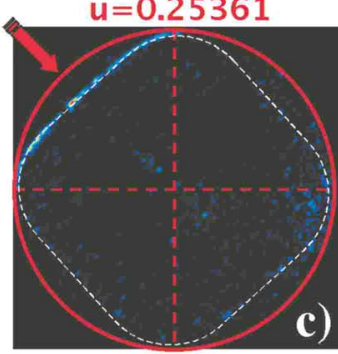

$\mathrm{u}=0.28315$

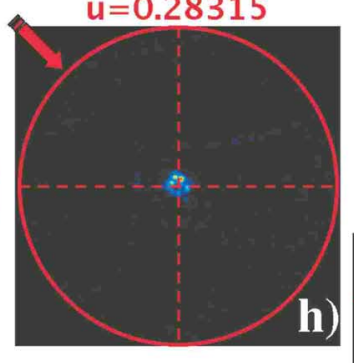

$u=0.26821$

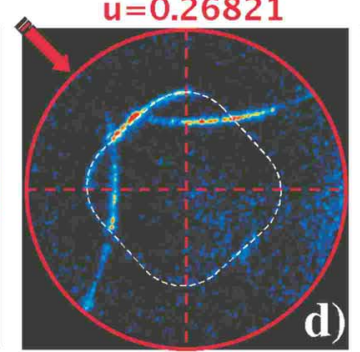

$\mathrm{u}=0.28323$

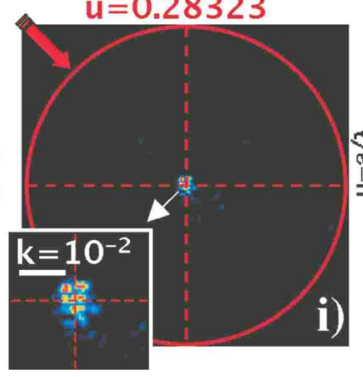

$\mathrm{u}=0.28094$
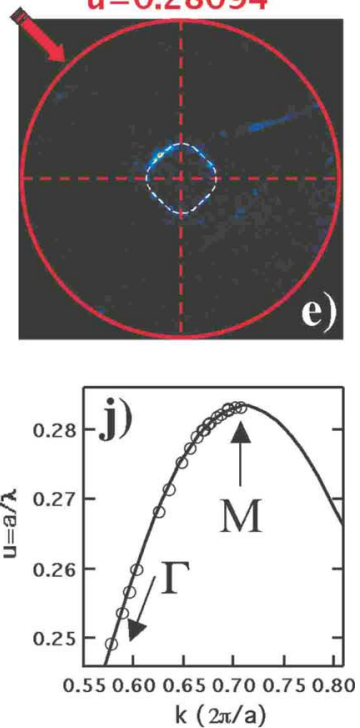

FIG. 2. (Color online) (a) Scanning electron micrograph of the SOI PhC tile and access waveguides with a zoom highlighting the PhC holes and the probe grating pattern in the inset. The dotted circle: area measured. (b)-(i) $k$-space images of the square lattice photonic crystal tiles near the first band edge for increasing reduced energy $u$. The red (dark gray) arrow: direction of the light excitation. The dashed white contours: theoretical equifrequency contours. The inset in (i): zoom of the center part of the $k$ space $\left(k\right.$ in units of $\left.\frac{2 \pi}{a}\right)$. (k) Corresponding experimental and theoretical dispersion band diagram. 
ciated with the EFS, $\vec{k}_{\text {out }}$ the wave vector of the scattered wave, and $\vec{G}$ one of the reciprocal vectors of the probe grating. ${ }^{6}$ As shown in Fig. 1(c), a proper choice of the geometrical parameters of the probe grating allows us to center the folded EFS in the middle of the light cone. In this case the probe grating has a square lattice symmetry with a lattice constant $\Lambda=2 a$ and $\vec{G}=\frac{2 \pi}{\Lambda} \vec{k}_{x}+\frac{2 \pi}{\Lambda} \vec{k}_{y}$ [Fig. 1(d)]. This principle of the folding of the $2 \mathrm{D}$ dispersion curve is experimentally carried out here to demonstrate an imaging below light cone, which is crucial to highlight, in the far field, the impact of the residual disorder.

Naturally, the probe grating presents a disturbance that should be as small as possible. It should be noted, however, that any techniques used to probe modes located below the light cone suffer similar limitations, e.g., the disturbance caused by the presence of a scanning near field tip ${ }^{7}$ or a hemispherical lens. ${ }^{8}$ Note that any structural defects can accomplish the information transfer in the far field by scattering a small part of the waves propagating below the light cone $^{3}$ as mentioned above. However, the measurement of the shape of the experimental EFS is smeared out by the intrinsic randomness of the defect assisted scattering process, especially near a band edge.

A $2 \mathrm{D}$ probe grating was directly etched into the surface of the $230 \mathrm{~nm}$ silicon layer used to form the PhC pattern [Fig. $2(\mathrm{a})]$. The $2 \mathrm{D}$ probe grating has to be etched shallow enough in order to avoid detrimental losses or the creation of a double PhC structure that would imply a strong modification of the EFS. The probe grating is composed of $70 \mathrm{~nm}$ wide and $20 \mathrm{~nm}$ deep etched lines. Note that such an intentionally etched probe is often even not required for low $e$-beam dose exposure: the intrinsic residual periodic errors resulting from the $e$-beam patterning process are sufficient to create a probe grating. We indeed also observed a 2D EFS folding induced by such residual gratings similar to the one recently observed in silicon wire waveguides. ${ }^{9}$

The small fraction of the field scattered in the far field by the shallow etched grating is then imaged either in the Fourier space located in the back-focal plane of the collecting lens (Fig. 2) or in the real-space-like standard microscope image (Fig. 3), as described in Ref. 10. In both cases the light comes from the same area of the $\mathrm{PhC}$ tile that is highlighted in Fig. 2(a) by the white dotted circle and selected by an intermediate iris positioned in the collecting path. The transition from real space to Fourier space is simply accomplished by adding one lens in the optical collecting path.

Figure 2 shows the evolution of the experimental EFS from the regime of self-collimation (where the light propagates without diffraction) toward the theoretical singular point at the top of the band. Lithographic tuning was used to span a wide frequency range, i.e., the top and bottom images correspond to two structures with different lattice constants, $a=430 \mathrm{~nm}$ and $a=400 \mathrm{~nm}$, respectively. The EFS calculated from the 2D plane wave expansion method (PWE) fits the shape and the position of the measured data very well, provided that the dispersion of the planar waveguide confining the mode in the $z$ direction is taken into account. Away from the top of the band, the EFS is mainly excited in the region where the group velocity is oriented along the forward
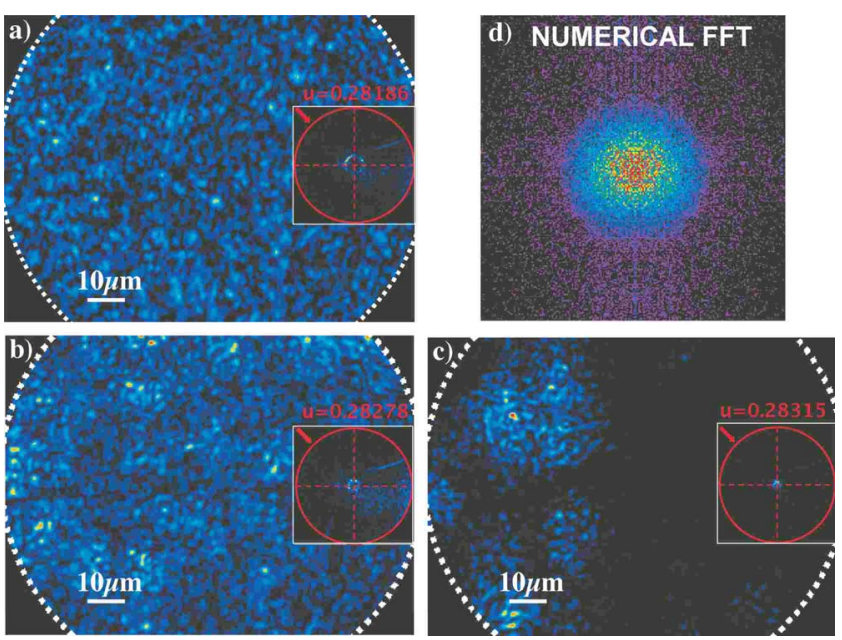

FIG. 3. (Color online) (a)-(c) Real-space image of the probed square lattice photonic crystal at different reduced energy $u$, with the probed area identical to the one in Fig. 2. Inset: corresponding optical Fourier space image. (d) Numerical fast Fourier transform of the real-space image shown in (a).

direction of propagation. The parts of the EFS that correspond to lateral propagation and to backward propagation require an additional scattering process to be excited. This is accomplished mainly by random residual defects.

When the excitation wavelength approaches the top of the band edge, the EFS evolves toward circles with decreasing radii before turning into a relatively wide spot at $u=a / \lambda$ $=0.28323$. As the EFS approaches the band edge, its excitation is more homogeneous, which is in line with the role played by the disorder. At the edge, the size of the spot is wider than the one resulting from a mode delocalized over the entire the $\mathrm{PhC}$ tile. In the corresponding real space [Figs. 3(a)-3(c)], we observe a transition from a field extended over all the PhC $(u=0.28186)$ to a field localized in distinct areas $(u=0.28315)$. It follows that the size of the spot in $k$ space is related to localization induced by the structural disorder and not to a field decay produced by pure dissipation such as the losses generated by the $2 \mathrm{D}$ probe grating. From the intensity decay along the light direction in the self-collimation regime, we deduce optical losses of $\sim 48 \mathrm{~cm}^{-1}$. The $k$-space broadening at the edge of the band $\delta k \simeq 0.005\left(\frac{2 \pi}{a}\right)$ is in agreement with the size of the localization areas $\delta x \simeq 12 \mu \mathrm{m}$. Strong localization of electromagnetic modes has been recently reported in one-dimensional (1D) disorder photonic crystal waveguides. ${ }^{11}$ Here, the transition from extended to localized modes is observed in a 2D geometry, which agrees with localized modes measured in a $2 \mathrm{D}$ open microwave cavity randomly filled with cylinders. ${ }^{12}$ In Fig. 2(j), the upper part of the $2 \mathrm{D}$ band, where the group velocity becomes theoretically low, is here related to localized modes as indicated by the linewidth broadening in $k$ space [Fig. 2(i)] and the real-space images [Fig. 3(c)].

In Fig. 3(a) we also show that the intensity pattern collected in real space has a complex specklelike structure, whereas the image in Fourier space exhibits the circular ring similar to the expected EFS shape. Although the investigated area is the same in both cases, the nature of the information 
is completely different. As revealed by the Fig. 3(d), a fast Fourier transform (FFT) of the real-space image cannot reproduce the pattern of the EFS. In real space, the relative phases between the different Bloch waves are lost as only the intensities of the components of the field are detected by the charge-coupled device (CDD) camera. In contrast, the imaging in the Fourier space gives a direct access to the different wave vectors that constitute the field. It confirms that the information about the relative phases is conserved. Thus, Fig. 3 is an enlightening illustration that emphasizes the essential physical role played by the phase in the imaging process and shows that in general a numerical Fourier transform of a real-space image is insufficient to retrieve the EFS.

In addition to the TE polarized EFS, we observe other features that reveal the presence of modes propagating along $x$ or $y$ for instance in Fig. 2(d). These features agree well with the folded TM polarized EFS calculated with 2D PWE. We attribute such a polarization conversion to the intrinsic asymmetry of the silicon on insulator planar waveguide and/or to the asymmetry induced by the tiny probe grating.

The measurement of the negative group-velocity part of the EFS in Fig. 2(b) demonstrates that $k$ vector with $|k|$ $\sim \frac{2 \pi}{a}$ is retrieved in the far field. It corresponds to the achievement of an effective numerical aperture as large as $\mathrm{NA}=3.5$. The demonstration of such a NA extension is appealing for the investigation of the spatial frequency spectrum of even more complex objects. We have considered here a structure whose entire set of spatial frequencies are located below the light cone. For objects that have also spatial frequencies inside the light cone, a clear distinction between the folded and unfolded spatial frequencies needs to be made. This distinction can be accomplished, for instance, by the acquisition of two images, one with the probe grating and the other without, and a proper analysis of the phase of the field. ${ }^{13}$

In conclusion, we have imaged, in both real space and Fourier space, a photonic band located below the light cone. By using a probe grating approach that enables one to investigate planar photonic structures free from intrinsic dissipation, the images near the photonic band edge highlight the localization effect induced by the structural disorder in a 2D geometry. From one side, this imaging is crucial to understand the physics of the wave localization in 2D disordered structures, as well as the limitation of nanophotonic structures imposed by their fabrication. From another side, the achievement of effective numerical apertures as large as 3.5, whose demonstration is based here on the ideal nature of $\mathrm{PhC}$ structures, paves the way for super-resolution imaging of more complex objects.

This project was supported by the EU European network of excellence Epixnet Grant No. IST-004525 and Funfox Grant No. IST-004582, the Swiss Quantum Photonics National Center for Competence in Research, and the COST action under Grant Nos. P11 and MP0702. The authors thank H. Zhang for the SEM images.
${ }^{1}$ T. F. Krauss and R. M. De La Rue, Nature (London) 383, 699 (1996).

${ }^{2}$ N. Le Thomas, V. Zabelin, R. Houdré, M. V. Kotlyar, and T. F. Krauss, Phys. Rev. B 78, 125301 (2008).

${ }^{3}$ R. Zengerle, J. Mod. Opt. 34, 1589 (1987).

${ }^{4}$ A. Sentenac, P. C. Chaumet, and K. Belkebir, Phys. Rev. Lett. 97, 243901 (2006).

${ }^{5}$ S. Durant, Z. Liu, J. M. Steele, and X. Zhang, J. Opt. Soc. Am. B 23, 2383 (2006).

${ }^{6}$ N. Le Thomas, R. Houdré, L. H. Frandsen, J. Fage-Pedersen, A. V. Lavrinenko, and P. I. Borel, Phys. Rev. B 76, 035103 (2007).

${ }^{7}$ R. J. P. Engelen et al., Nat. Phys. 3, 401 (2007).

${ }^{8}$ M. Galli, D. Bajoni, M. Patrini, G. Guizzetti, D. Gerace, L. C.
Andreani, M. Belotti, and Y. Chen, Phys. Rev. B 72, 125322 (2005).

${ }^{9}$ J. Jágerská, N. Le Thomas, R. Houdré, J. Bolten, C. Moormann, T. Wahlbrink, J. Čtyroký, M. Waldow, and M. Först, Opt. Lett. 32, 2723 (2007).

${ }^{10}$ N. Le Thomas, R. Houdré, M. V. Kotlyar, D. O'Brien, and T. F. Krauss, J. Opt. Soc. Am. B 24, 2964 (2007).

${ }^{11}$ J. Topolancik, B. Ilic, and F. Vollmer, Phys. Rev. Lett. 99, 253901 (2007).

${ }^{12}$ D. Laurent, O. Legrand, P. Sebbah, C. Vanneste, and F. Mortessagne, Phys. Rev. Lett. 99, 253902 (2007).

${ }^{13}$ N. Le Thomas, R. Houdré, M. V. Kotlyar, and T. F. Krauss, Phys. Rev. B 77, 245323 (2008). 